\title{
Recent Trends in Energy-Efficient Clustering in WSNs
}

\author{
Md.Faruqul Islam \\ Apex Institute of \\ Engg.\&Tech. \\ Jaipur,Rajasthan
}

\author{
Yogesh Kumar \\ Apex Institute of \\ Engg.\&Tech. \\ Jaipur,Rajasthan
}

\author{
Saurabh \\ Maheshwari \\ Govt.Women \\ Engg.College
}

\author{
Ajmer,Rajasthan \\ Neeti Jain \\ Govt.Engg.College \\ Ajmer,Rajasthan
}

\begin{abstract}
Tremendous growth has been observed in the use of Wireless sensor networks (WSNs) in the last decade. WSNs behave like an interface between the virtual and physical worlds. Sensor nodes sense the changes in external environment and send the data to other nodes in the network or the sink, also called Base Station (BS). The performance of the network gets affected due to limited battery lifetime of sensor nodes. Many clustering and power management protocols have been specifically designed for WSN to reduce energy consumption. Improvements are being made day by day in the basic clustering protocols. In this paper, a survey of the recent clustering protocols in WSNs has been presented and a comparison has been done of various protocols.
\end{abstract}

\section{Keywords}

Wireless Sensor Networks, Clustering, cluster head $(\mathrm{CH})$, sensor nodes.

\section{INTRODUCTION}

WSNs are an emerging technology that consists of a large number of nodes. A sensor node is a low cost and low power electronic device that is capable of detecting changes in physical environmental conditions. These nodes communicate with each other either directly or through intermediate nodes for collecting and transferring information to the base station (BS). The location of BS is generally fixed and it is located far away from the nodes. These sensor nodes work together to form a wireless network that can be used for many applications such as monitoring physical events, for example environmental monitoring, battlefield surveillance, disaster relief, target tracking, etc.

Sensor nodes in WSN consists of four components [1]: a sensor which performs sensing according to a particular application, a radio transceiver that performs radio transmission and reception, a microcontroller which is used for processing of data and a battery for providing energy.

One of the most crucial problems in WSN is the limited battery power of each node, with no form of recharging after deployment in the network. Many protocols have been proposed for energy saving in WSNs. Most of the algorithms proposed for WSNs focus on increasing the lifetime of the network by trying to reduce the

energy consumption [1].It has been found that clustering of nodes in WSNs is an effective program of energy conservation [2]. In

WSN, it is used to minimize the number of nodes that take part in long distance data transmission to a Base Station, thus lowering the total energy consumption of the system [3].

Each clustering algorithm is divided into 3 phases: cluster head $(\mathrm{CH})$ selection, the setup phase, and steady state phase.
The main point in clustering algorithms is the cluster head selection.

\section{CLUSTERING SCHEME OVERVIEW}

\subsection{What Is Clustering?}

In clustering, the sensor nodes in a WSN which are located geographically adjacent to each form a group and come in the same cluster following some set of rules. Inside a cluster structure, sensor nodes serve either as a cluster head or a cluster member [4]. A cluster head acts like a local coordinator for its cluster and performs functions such as transmission among different clusters, data forwarding, etc. Data collected from different nodes is aggregated at the cluster head which is then forwarded to the base station as shown in Figure 1. Advantages of clustering are: (1) it reduces energy consumption by improving bandwidth utilization (2) reduces wasteful consumption of energy by reducing overhead. Most of the algorithms try to maximize the network lifetime by equalizing energy consumption between nodes and by distributing the load between different nodes from time to time [5]. The cluster head is changed during the reformation of a cluster along with the members in it.

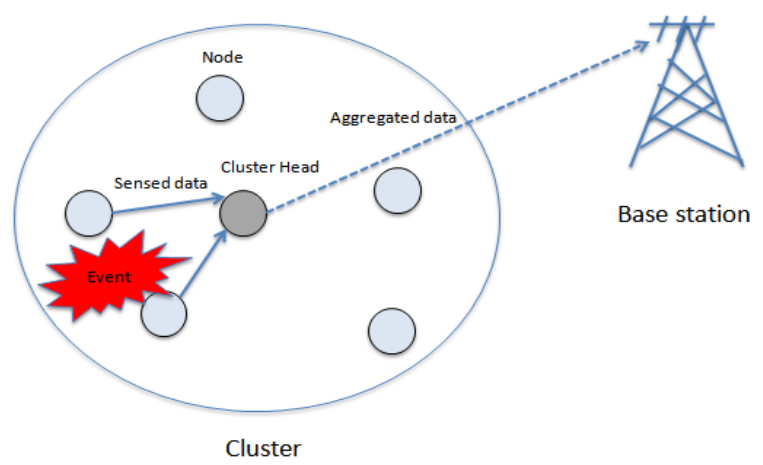

Fig1: Event detection at the cluster head [4]

\subsection{Why Do WSN Require Clustering?}

Clustering is used in WSN when there are a large number of nodes involved. It increases system capacity by reusing the network's resources. Two nearby clusters cannot use the same frequency or code set [6,7]. Cluster heads enhance the performance in case of routing as they act like a virtual backbone in intercluster routing. Clustering in WSNs is very difficult due to the inherent characteristics which differentiate these networks from other wireless networks such as mobile ad hoc networks or cellular networks [8]. It is difficult to distinguish the sensed data came from which node due to large number of nodes. Communication within one cluster as well as between different clusters can take place as combination of single hop and multi- hop as illustrated in fig. 2. In single hop communications, each sensor node directly 
communicates with the BS. While in multi- hop communications, nodes route their data over several hops until the data reaches $\mathrm{BS}$; due to limitations in their transmission range.

\subsection{Types of Clustering}

Clustering algorithms differ with respect to the metrics they use for cluster control such as energy, lifetime calculations, hops, distance from the cluster head and also the type of controls such as centralized or distributed [9].

\subsubsection{Hierarchical clustering (Connectivity based clustering)}

In Hierarchical clustering, network is split into several clustered layers [10], and data travels from a lower clustered layer to a higher clustered layer. Data is first aggregated but as it moves from one node to another it covers greater distances that helps the data to reach the BS faster, thus reducing travel time and latency [11].

\subsubsection{Data centric clustering}

Data centric clustering is designed to find shortest distance between pairs of nodes [12]; it aggregates data and sends it from different recourses to a destination using named data. Since assigning global identifiers to every sensor nodes in a WSN may not be feasible due to the huge number, nodes are addressed by their locations, proximity, or capability rather than a globally unique identifier.

\subsection{Challenges of Clustering}

WSNs present vast challenges in terms of implementation. There are several key attributes that designers must carefully consider, which are of particular importance in wireless sensor networks.

- Cost of Clustering

- Selection of Cluster heads and Clusters

- Real-Time Operation

- Synchronization

- Data Aggregation

- Repair Mechanisms

- Quality of Service (QoS)

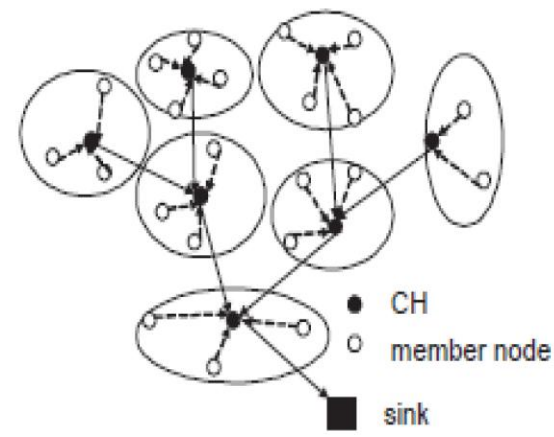

Fig 2: Network illustrating single hop and Multi-hop routing in WSN.

\section{CLUSTERING PROTOCOLS}

During last few years many clustering algorithms have been proposed for Wireless Sensor Networks as an efficient way for balancing the energy consumption and prolonging the lifetime of the networks [13]. Here we mention some of the recent clustering algorithms.

\subsection{Equalized Cluster Head Election Routing Protocol (ECHERP)}

ECHERP [14] uses an energy efficient mechanism to select a node as the cluster head. ECHERP considers the current and the estimated future residual energy of the nodes, along with the number of rounds that can be cluster heads in order to maximize the network lifetime. The protocol computes the energy consumed using the Gaussian elimination algorithm in order to minimize the overall network energy consumption at every single round. The selection of $\mathrm{CH}$ is done on the bases of minimization of total energy consumption; the node that does so is elected as the $\mathrm{CH}$ rather than the node having the highest energy left, as in many other protocols. ECHERP also adopts a multi-hop routing scheme to transfer fused data to the base station

\subsection{Distance based Cluster Head Selection}

This protocol [15] performs better in terms of network lifetime by balancing the energy load among all the nodes. This algorithm selects cluster head among some of the wireless sensor nodes based on net distance with base station. This technique aims to increase the lifetime of the whole network, and to increase the number of nodes, which will remain alive for the maximum period of time.

\subsection{Cost Based Energy Balanced Clustering and Routing Algorithm(CEBCRA)}

CEBCRA is distributed algorithm which consists of three phases namely Broadcast node, $\mathrm{CH}$ selection and Data Transfer. CEBCRA selects $\mathrm{CHs}$ amongst the normal sensor node using a weight function of the residual energy and the number of neighbors of a sensor node [16]. Then all non-CH sensor nodes join a $\mathrm{CH}$, which has maximum cost value within its communication range. The cost function is the composite measure of residual energy of the $\mathrm{CH}$, its distance to base station and also the distance from the sensor node to the $\mathrm{CH}$. For multi-hop routing, a $\mathrm{CH}$ needs to relay the data to the BS through other $\mathrm{CHs}$. Therefore, the other $\mathrm{CHs}$ are treated as relay nodes. So, a $\mathrm{CH}$ needs to find the best neighbor $\mathrm{CH}$ (relay node) for data routing such that energy consumption is minimum.

\subsection{Battery Level Aware Clustering(BLAC)}

BLAC considers the battery level along with density or degree of a node to choose the cluster-head. It aims at increasing the lifetime, thus keeping all the nodes alive to perform a specific task. Simulation results proved that BLAC increases the full network lifetime by three times than the traditional clustering schemes through balancing energy consumption of nodes and it gives high data ratio [17]. BLAC has four variants; BLACbg uses battery level and degree of a node; BLAC-bs combines the battery level and density of a node. BLAC-rg and BLAC-rs run in two steps. They reduce graphs before calculating the clusters. Each of these variants have their particular features and used according to the specific application.

\subsection{Regional Energy Efficient Cluster Heads based on Maximum Energy Routing Protocol (REECH-ME)}

The basic aim of this protocol [18] is to maximize the lifetime of a network and it especially focuses on its stability period. In this, a node having the maximum energy in a region is selected as a Cluster Head $(\mathrm{CH})$ of that region for a particular round and number of the $\mathrm{CHs}$ in each round remains the same. 
Thus, this scheme enhances the desired attributes, i.e. minimum energy consumption, maximum stability period, better lifetime and throughput allot.

\subsection{Energy efficient heterogeneous clustered scheme (EEHC)}

EEHC [19] works on three types of sensor nodes normal nodes, advanced nodes and super nodes. There are $\mathrm{N} \times \mathrm{m} \times \mathrm{mo}$ number of super nodes used with $\beta$ times more energy than the normal node. Where $\mathrm{N}$ represents the total number of nodes $\mathrm{m}$ and $\mathrm{mo}$ are the fraction of the total number of nodes. The rest $\mathrm{N} \times \mathrm{m} \times(1-\mathrm{mo})$ number of advanced nodes which are equipped with $\alpha$ times more energy than the normal nodes; the remaining $\mathrm{N} \times(1-\mathrm{m})$ number of normal nodes. EEHC is more effective in prolonging network lifetime as compared to LEACH

\subsection{Enhanced Developed Distributed Energy-Efficient Clustering (EDDEEC)}

EDDEEC is an adaptive energy aware protocol. It dynamically varies the probability of nodes to become a $\mathrm{CH}$ in a balanced and efficient way in order to distribute equal amount of energy between sensor nodes. EDDEEC worked on three types of heterogeneous nodes, for probabilistic selection of $\mathrm{CH}$ a routing algorithm is used. [20]. EDDEEC prolongs network lifetime, longer stability period and increased number of messages sent to base station as compared to DEEC [21], DDEEC [21], and EDEEC [21].

\subsection{Balanced Energy Efficient Network Integrated Super Heterogeneous Protocol (BEENISH)}

BEENISH worked on four types of heterogeneous nodes as normal nodes, advanced nodes, super nodes and ultra-super nodes. In BEENISH, CH is selected based on ratio of residual energy of a node and average energy in the network field [22]. BEENISH prolongs network lifetime, longer stability period and increased number of messages sent to base station as compared to DEEC, DDEEC, and E-DEEC.

\subsection{Energy Efficient Clustered Chain Based Power Aware Routing Protocol (EECCPAR)}

The main goal of this protocol is to enhance the lifetime of the network and reduce the communication cost by evenly distributing the energy load among all sensor nodes [23]. This protocol divides the network into clusters and then constructs chain among cluster members and also among CHs. During data transmission $\mathrm{CHs}$ introduces three Thresholds i.e. MIN, MAX and Change Factor. The performance of this protocol is better then PEGASIS. This protocol doesn't suffer from clustering overhead, since clustering is not performed in each phase.

\subsection{Event Driven Hierarchical Cluster based Protocol}

This is an energy efficient hierarchical routing technique [24] in which cluster heads are selected based on the prediction of transmission energy via shortest possible distance to the base station for transmitting the event driven information. In this clusters of the sensor nodes are created geographically, rotate the role of $\mathrm{CH}$, and optimize the $\mathrm{CH}$ selection by the help of prediction of energy used for transmission in every rounds of simulation, and when event occur $\mathrm{CH}$ aggregates event data before transmitting it to the BS. The concept of hierarchical routing technique can be effectively used along with event driven routing to designed energy efficient routing protocol in WSN. In this algorithm, the clusters are geographically formed into different sizes to see how it could affect the network lifetime of WSN.

\subsection{Distributed hierarchical agglomerative clustering (DHAC)}

Hierarchical agglomerative clustering (HAC) [25] is a conceptually and mathematically simple clustering approach which uses four clustering methods, CLINK, SLINK, UPGMA, and WPGMA. All of these methods comprise three common key steps: obtain the data set, build the similarity matrix, and execute the clustering algorithm. Based on the concept of HAC, DHAC method was proposed for distributed environments by improving the HAC algorithms. The main idea behind DHAC is that a node only needs one-hop neighbor knowledge to build clusters. To apply the DHAC algorithm in WSNs, a bottom-up clustering approach is followed using simple six steps. Firstly, the qualitative connectivity data is obtained as input data set for DHAC. Secondly, the similarity matrix is built. Thirdly, the similar nodes are grouped together by executing the distributed clustering algorithm. The last three steps are cutting the cluster tree with the threshold, merging the smaller cluster, and electing the CHs. the performance of DHAC is much better than LEACH. The clustering dissipated energy of DHAC is about 4 times less than that of LEACH which predicates that DHAC can achieve much high reliability and efficiency in energy consumption at 300 rounds timing.

\subsection{Density-based Energy-efficient Clustering Heterogeneous Algorithm (DECHA)}

In this algorithm, the election probability of nodes to become cluster heads is evaluated [26]. With regard to the probability, there is density referring to the position information of a node, and together with its energy capacity serve as primary weighted metrics. Further evaluation is done for a better selection of cluster heads. In DECHA, the position information of nodes, define the density of each node as the number of its neighbor nodes is considered, and together with the energy capacity, regard it as an important evaluation metric for electing candidate CHs. DECHA also sets further adjustments to seek more proper $\mathrm{CHs}$, thus promote both lifetime and energy-efficiency.

\subsection{Low Energy Time Based Clustering Technique}

This clustering technique [27] is based on the residual energy of nodes to maximize the network lifetime. Each node decides to be $\mathrm{CH}$ depending on its residual energy and the average energy of network's nodes. As $\mathrm{CH}$ performs data aggregation in order to reduce the number of packets to be transmitted to the Base Station (BS), the choice of $\mathrm{CH}$ must be optimal so that the compressed data at $\mathrm{BS}$ is reliable. 
TABLE 1: Comparison on the basis of LND for Various Algorithms

\begin{tabular}{|c|c|c|c|c|}
\hline \multirow[t]{2}{*}{ Algorithm } & \multicolumn{2}{|c|}{$\begin{array}{l}\text { Last node dead } \\
\text { time(LND) }\end{array}$} & \multirow{2}{*}{$\begin{array}{l}\text { No. of } \\
\text { nodes }\end{array}$} & \multirow{2}{*}{ Area } \\
\hline & $L E A C H$ & $\begin{array}{c}\text { Proposed } \\
\text { Algorithm }\end{array}$ & & \\
\hline ECHERP & $\begin{array}{l}\text { After } \\
110^{\text {th }} \\
\text { round }\end{array}$ & $\begin{array}{c}\text { After } \\
145^{\text {th }} \\
\text { round }\end{array}$ & 500 & $\begin{array}{c}100 \mathrm{~m} \times \\
100 \mathrm{~m}\end{array}$ \\
\hline DECHA & $\begin{array}{l}1195^{\text {th }} \\
\text { round }\end{array}$ & $\begin{array}{l}4924^{\text {th }} \\
\text { round }\end{array}$ & 100 & $\begin{array}{c}500 \mathrm{~m} x \\
500 \mathrm{~m}\end{array}$ \\
\hline $\begin{array}{l}\text { REACH- } \\
\text { ME }\end{array}$ & $\begin{array}{l}1500^{\text {th }} \\
\text { round }\end{array}$ & $\begin{array}{l}2500^{\text {th }} \\
\text { round }\end{array}$ & 100 & $\begin{array}{c}100 \mathrm{~m} \mathrm{x} \\
100 \mathrm{~m}\end{array}$ \\
\hline DHAC & $\begin{array}{c}90 \% \\
\text { nodes } \\
\text { dead till } \\
585^{\text {th }} \\
\text { second }\end{array}$ & $\begin{array}{c}90 \% \\
\text { nodes } \\
\text { dead till } \\
1120^{\text {th }} \\
\text { second }\end{array}$ & $\begin{array}{c}\text { Total time } \\
\text { interval } \\
=1200 \mathrm{sec} \\
\text { Node } \\
\text { Number } \\
100\end{array}$ & $\begin{array}{c}100 \mathrm{mx} \\
100 \mathrm{~m}\end{array}$ \\
\hline
\end{tabular}

\section{CONCLUSION}

In this paper, a detail survey of the recent clustering algorithms is presented and discussed for energy constrained WSNs. Protocols discussed in this paper offer a considerable improvement over traditional clustering; however there is still a lot of work to be done. Each protocol has its own merits and demerits which makes them appropriate for specific applications. The power of the node needs to be used in a more secure and efficient way. A brief comparison of some of the protocols has been summarized in Table 1. CEBCRA protocol provides improvement over traditional protocol in terms of network lifetime.

The clustering protocols can be made more effective in future by considering metrics related to QoS and time constraints. Further, security needs to be incorporated in them in order to protect data from eavesdroppers.

\section{REFERENCES}

[1] Chaurasiya S.K., Pal T, Bit S.D, "An Enhanced EnergyEfficient Protocol with Static Clustering for WSN". International Conference on Information Networking (ICOIN), 2011 Kuala Lumpur, Malaysia, on page(s): 58 -63 .

[2] Ismail Olalekan Eleburuike; Soetan Simeon Adekunle, "Energy Efficient Wireless Sensor Network Using Hierarchical Routing Technique"available:http:/www.bth.se/fou/cuppsats.nsf/ all/6b6f6b72800ae835c1257749004a55a8/\$file/Final\%2 0Report_ReportIsmail\%26Simeon.pdf Blekinge Institute of Technology, Karlskrona, Blekinge, Sweden, 2010.

[3] J. Yang, D. Zhang, "An Energy-Balancing Unequal Clustering Protocol for Wireless Sensor Networks". Information Technology Journal, 2009, 57-63.

[4] X. Zhu, L. Shen, and T. S. P. Yum, "Hausdorff clustering and minimum energy routing for wireless sensor networks," in Proceedings of the 18th Annual IEEE International Symposium on Personal, Indoor and Mobile Radio Communications (PIMRC '07), pp. 1-7, September 2007.

[5] Bouabdallah, M. E. Rivero-Angeles, and B. Sericola, "Continuous monitoring using event-driven reporting for cluster-based wireless sensor networks," IEEE Transactions on Vehicular Technology, vol. 58, no. 7, pp. 3460-3479, 2009.

[6] Boukerche, H. A. B. F. Oliveira, E. F. Nakamura, and A. A. F. Loureiro, "Localization systems for wireless sensor networks," IEEE Wireless Communications, vol. 14, no. 6, pp. 6-12, 2007.

[7] R. Rajagopalan and P. K. Varshney, "Data aggregation techniques in sensor networks: a survey," IEEE Communications Surveys \& Tutorials, vol. 8, no. 4, pp. 48-63, 2006.

[8] M. C. M. Thein and T. Thein, "An energy efficient clusterhead selection for wireless sensor networks," in Proceedings of the UKSim/AMSS 1st International Conference on Intelligent Systems, Modelling and Simulation (ISMS '10), pp. 287-291, January 2010.

[9] Venkataraman, G.; Emmanuel, S.; Thambipillai, S.; "DASCA: A Degree and Size based Clustering Approach for Wireless Sensor Networks" 2nd International Symposium on Wireless Communication Systems, 2005, Siena, Italy. Page(s): 508 - 512.

[10] Xinyuan Zhao; Neng Wang, "An unequal layered clustering approach for large scale wireless sensor networks", 2nd International Conference on Future Computer and Communication (ICFCC), 2010, Wuhan, China, on page(s): 750 -756.

[11] W. Li, G. Chen, "Energy-Efficient Clustering Algorithm in Wireless Sensor Network", International Conference in IET on pp. 1-4, November, 2006.

[12] Qinghai Gao, Junshan Zhang; Larish, B.; "Energy Balancing in Coalition-Based Multi-Hop Wireless Sensor Networks" Military Communications Conference, 2006. Washington, DC, USA. Page(s): $1-7$.

[13] Neeti Jain, Prakriti Trivedi, "Evolution of Advanced Clustering methods in WSN: A Review",IJCA,Volume 56,No.11,2012.

[14] Stefanos A. Nikolidakis, Dionisis Kandris , Dimitrios D. Vergados and Christos Douligeris, "Energy Efficient Routing in Wireless Sensor Networks Through Balanced Clustering"Algorithms,2013,pp.29-42.

[15] Bhawnesh Kumar, Vinit Kumar Sharma, "Distance based Cluster Head Selection Algorithm for Wireless Sensor Network" International Journal of Computer Applications (0975 - 8887) Volume 57- No.9, November 2012

[16] Pratyay Kuila and Prasanta K. Jana, “An Energy Balanced Distributed Clustering and Routing Algorithm for Wireless Sensor Networks," IEEE International Conference on Parallel, Distributed and Grid Computing, pp. 220-225, 2012.

[17] Tony Ducrocq, Nathalie Mitton, Michael Hauspie, "Energy-based Clustering for Wireless Sensor Network 
Lifetime Optimization" WCNC .Wireless Communications and Networking Conference (2013)

[18] A. Haider, N. Javaid, N. Amjad, A. A. Awan, A. Khan, N. Khan, "REECH-ME: Regional Energy Efficient Cluster Heads based on Maximum Energy Routing Protocol for WSNs" arXiv:1307.7052v1 [cs.NI] ,26 Jul 2013.

[19] Dilip Kumar, Trilok C. Aseri, R.B. Patel, EEHC: Energy efficient heterogeneous clustered scheme for WSNs, ELSEVIER, Computer Communications, pp: 662-667, 2009.

[20] N. Javaid, T. N. Qureshi, A. H. Khan, A. Iqbal, E. Akhtar, M. Ishfaq, "EDDEEC: Enhanced Developed Distributed Energy-Efficient Clustering for Heterogeneous Wireless Sensor Networks" International Workshop on Body Area Sensor Networks (BASNet2013),March 2013.

[21] Padmavati, T.C. Aseri, "Heterogeneous cluster based routing protocols in wireless sensor Network- a Survey" International Journal of Research in Advent Technology, Volume 1, Issue 4, November 2013

[22] T.N. Quereshi, N.Javaid, A.H. Khan,A. Iqbal, E.Akthar, M.Ishfaq, "BEENISH: Balanced Energy Efficient Network Integrated Super Heterogeneous Protocol for
Wireless Sensor Networks," International workshop on Body Area Sensor Networks, pp: 914-919, 2013.

[23] Parendra Kumar Singh, "Energy Efficient Clustered Chain Based Power Aware Routing Protocol for Wireless Sensor Networks" Thesis, Department of Computer Science and Engineering National Institute of Technology Rourkela, 2013

[24] S Taruna, Megha R. Tiwari, “ Event Driven Hierarchical Cluster based Routing Protocol for Wireless Sensor Network" International Journal of Advanced Research in Computer Science and Software Engineering,Volume 3, Issue 4, April 2013.

[25] Chung-Horng Lung, Chenjuan Zhou, "Using hierarchical agglomerative clustering in wireless sensor networks: An energy-efficient and flexible approach" IEEE "GLOBECOM" 2008 proceedings.

[26] Zhanyang Xu, Yue Yin, Jin Wang, “An Energy-efficient Clustering Routing Algorithm for Heterogeneous Wireless Sensor Networks" NGCIT 2013, ASTL Vol. 27, pp. $85-93,2013$.

[27] Ouadoudi Zytoune1, Driss Aboutajdine, “A Low Energy Time Based Clustering Technique for Routing in Wireless Sensor Networks" American Journal of Sensor Technology, 2014, Vol. 2, No. 1, 1-6. 